\title{
OBSERVABLE CONSEQUENCES OF TRIAXIAL HALOS
}

\author{
James Binney \\ Department of Theoretical Physics, \\ Keble Road, Oxford, OX1 3NP, \\ England.
}

\begin{abstract}
Heavy halos are probably not axisymmetric. During disk formation, the visible parts of disk galaxies are likely to have aligned their principal axes with those of the surrounding halos, but it is less clear that early-type galaxies are aligned with their halos. Triaxial halos may possibly sustain, or even excite, the warps often seen in the outer parts of disks. Polar rings and ripples around elliptical galaxies provide promising probes of the triaxiality of halo potentials.
\end{abstract}

\section{Are Halos Triaxial and are they Aligned with their Galaxies?}

Numerical simulations have shown that the collapse and virialization of a cloud of stellar objects from a wide variety of spherical (Barnes, Goodman \& Hut 1985, Merritt \& Aguilar 1985), and non-spherical (Binney 1976, Aarseth \& Binney 1978, Miller \& Smith 1981, Wilkinson \& James 1982) initial configurations leads to the formaton of a triaxial system. Furthermore, encounters of stellar systems, especially nearly head-on encounters, often yield triaxial remnants (White 1979, Gerhard 1981, 1983, Negroponte \& White 1982). Hence if, as seems overwhelmingly probable, massive halos formed by either the collisionless collapse of single systems or the merging of two or more collisionless systems, many halos should be markedly triaxial, and it is interesting to seek observational implications of this triaxiality.

The observational consequences of a halo's triaxiality depend very much on whether the halo's principal axes are aligned with those of the embedded galaxy. A prima facie argument against such alignment may be developed from the picture of galaxy and halo formation described by Gunn (1982). In this picture the galaxy's spheroidal component collapses first, and the disk and halo later, by way of infall. Hence the angular momentum and principal axes of the spheroid would have been determined earlier, and by sampling the ambient tidal field at smaller radii, than the principal axes of the halo and the angular momentum of the protodisk material. Consequently, we would not expect the original angular momentum vector of the 
spheroid to be parallel to that of the halo/disk. Indeed we might even anticipate a tendency to anti-alignment, since the spheroid will have acquired its angular momentum by exchange with the halo/disk. Hence elliptical galaxies, which are in this picture simply unreconstructed spheroids, are not expected to be aligned with their halos.

This argument does not apply to disk galaxies, since the spheroid of a disk galaxy must have been profoundly modified by the formation of the disk (Barnes \& White 1984, Binney \& May 1985). Estimates of the efficiency of angularmomentum acquisition from tidal interactions (Peebles 1969, Efstathiou \& Jones 1979) suggest (Binney \& Silk 1978, Fall \& Efstathiou 1980) that the disks are made of gas left over from halo formation. Hence one expects the protodisk material to have had similar angular momentum per unit mass to that of the halo. Furthermore, any mismatch in the directions of their angular momenta should have been ironed out as the protodisk material spiralled through the halo towards the central spheroid. In Oxford we have recently analyzed the response of an axisymmetric spheroid to the steady accretion of material which is endowed with angular momentum about an axis that is skew to the spheroid's symmetry axis, and have concluded (Binney \& May 1985) that the spheroid and any embedded disk would be dragged round into alignment with the angular momentum of the accreting material exactly as if it were a rigid body. Hence we expect disk galaxies to be aligned with their halos.

If, as White \& Rees (1978) have argued, halos formed first, and both spheroids and the disks of galaxies formed from material that fell into preexisting halos, every galaxy would from the beginning have taken its cue from the dominant halo field, and galaxy and halo would always be aligned.

\section{Halos and Warps}

Toomre (1983) has reviewed the possible connection between halos and warped disks, and Sparke (1984a) has recently given a thorough discussion of the dynamics of self-gravitating disks in axisymmetric potentials. The simplest way in which a halo can contribute to the longevity of a warp, is by making the gravitational potential of the whole system more nearly spherical; the wind-up time of a warp is approximately proportonal to the inverse of the ellipticity of the equipotential surfaces. Tubbs \& Sanders (1979) have argued that the best observed warps could last several billion years if the galaxies in which they occur have plausible massive halos.

Petrou (1980) has proposed a refinement of this idea by suggesting that the ellipticities of massive halos may frequently be increasing functions of radius. If this were so, the ellipticities of the overall galactic potentials might increase outwards fast enough to make the precession frequencies of disk stars roughly radiusindependent notwithstanding the steep outward decrease in the overall orbital frequencies. If halos are constructed like massive elliptical galaxies, an outward 
increase in their ellipticities is certainly to be expected (Di Tullio 1979).

Another simple way in which a halo could sustain a warp, is by being misaligned with the galaxy (Dekel \& Shlosman 1983, Toomre 1983, Marthur 1984). Deep inside the galaxy, gas will settle to the galaxy's equatorial plane, while far out, gas will follow a principal plane of the halo. The transition between these two regimes would be sharp and would be interpreted as the onset of a warp. If halos were expected to be misaligned with their embedded galaxies, this would be an attractive proposal. However, as explained above, one wonders whether such misalignment is truly possible, since the halo, through the agency of the protodisk material, should long ago have reoriented the galaxy parallel to the halo axes.

The warp theories described so far concern ways in which even an axisymmetric halo could increase the useful life of a warp that may not be caused by the halo itself. If the halo is triaxial, there are two ways in which the halo could generate a warp $a b$ initio:

1) Binney $(1978,1981)$ pointed out that orbits in a fundamental plane of a triaxial halo are unstable to vertical perturbations whenever the frequency at which orbiting stars pass density maxima is equal to twice the natural frequency of $z$ oscillations for these stars. In a non-rotating potential, this frequency condition is satisfied at all radii for orbits in the plane perpendicular to the intermediate axis of the potential (Heiligman \& Schwarzschild 1979). In a rotating potential the condition is satisfied $(i)$ on all retrograde orbits in an annulus that is located well inside the corotation radius of the potential, and (ii) on prograde orbits in an annulus that lies beyond corotation. When gas that is spiralling in from infinity along a fundamental plane of a triaxial potential reaches the outer bound of such an unstable zone, it will tend to lift from the plane. Heisler Merritt \& Schwarzschild (1982) and Mulder (1984) have followed the sequences of closed orbits onto which gas in an otherwise empty galaxy would then move. The sequence of closed retrograde orbits tends ultimately to align with the plane perpendicular to the longest axis of the potential, while orbits of the prograde sequence become self-intersecting near corotation; presumably gas would drop back onto the plane once the self-intersecting orbits were reached. Observationally this might mark the onset of a warp.

2) Bertin \& Mark (1980) have shown that corrugations in a thin cold disk that rotates through a slowly rotating axisymmetric halo, can grow unstably. A crude physical picture of this instability is as follows. We imagine the halo to be made up of a large number of streams of stars with the same velocity $v$. Each piece of the disk tries to focus these streams, much as in the standard picture of dynamical friction, a massive body focusses streams of background stars. When the disk is flat and axisymmetric, each element of the disk focusses upward- and downwardmoving streams to the same degree, so no net vertical forces are generated. But if some piece of the disk is moving up, it focusses the upward-moving halo stars more successfully than the downward-moving ones, and hence produces a net wake above 
it. This wake draws the offending section of the disk up still more, and overstable oscillations can set in.

Toomre (1983) has questioned the ability of these instabilities to generate warps in galactic disks, by noting that in a self-gravitating disk, corrugation waves have non-zero group velocities. Hence such waves carry energy from any region of overstable oscillations, and this will severely attenuate, or even eliminate, the growth of oscillations, unless something reflects the outgoing corrugation waves back into the unstable region. Recently Sparke (1984b) has investigated the effect of including realistic self-gravity on the Mathieu instability discussed by Binney. She finds that for realistic parameters, self-gravity eliminates the instability otherwise generated by a prograde halo, but the instability generated by a retrograde halo is only weakened by self-gravity.

Sparke interprets the survival into the self-gravitating regime of the instability associated with a retrograde halo in terms of a WKBJ analysis of tightly wound corrugation waves. This shows that the triaxiality of the halo causes leading waves, which propagate in one direction, to be converted into trailing waves, which propagate in the opposite direction, and vice-versa, thus enabling the wave energy to be fed again and again through the region of overstability. This is an interesting result with implications that extend beyond the Mathieu instability. Indeed it might even enable fuzzy-edged disks in triaxial halos, unlike similar disks in axisymmetric systems (Hunter \& Toomre 1969), to admit discrete bending modes, and thus support long-lasting warps even in the absence of an exciting mechanism.

\section{Polar Rings}

The last few years have seen several observational studies of "spindle" galaxies (Schechter \& Gunn 1978, Schweizer, Whitmore \& Rubin 1983, Schechter et al.1984, Schechter, Ulrich \& Boksenberg 1984). These systems consist of a central galaxy encircled by an bright annulus whose apparent major axis nearly coincides with the apparent minor axis of the central galaxy. The ratio of the outer and inner radii of the annulus can be as great as 3 . The annuli often seem to be reasonably flat. In every case studied, spectroscopic and photometric observations have revealed the central galaxy to be a nearly edge-on S0 galaxy, and the bright annulus to consist of gas and young stars. Since the annuli, or rings, of these galaxies extend well beyond the visible part of the underlying galaxies, they provide excellent probes of halos.

There are two ways in which rings may place constraints on halos:

1) Dissipation tends to push gas towards closed loop orbits. In an axisymmetric potential, two types of closed orbit are possible; $(i)$ orbits in the potential's equatorial plane, and (ii) orbits that pass through the potential's symmetry axis. Orbits within the equatorial plane are stable in the sense that if a particle on 
such an orbit is deflected through a small angle, it subsequently librates about its original closed trajectory. Polar orbits, by contrast, are neutrally unstable; a small-angle deflection of a particle on one of these orbits causes the particle to diverge from its original trajectory at an approximately constant rate. In a slowly rotating triaxial potential such as that generated by a triaxial halo, three types of closed orbit are possible; $(i)$ stable loop orbits that circulate about the potential's shortest axis; (ii) stable orbits that at low energies circulate about the potential's long axis, and (iii) unstable orbits that circulate around the potential's middle axis.

Gas that is injected into a halo on a non-closed orbit might be expected eventually to move to a stable closed orbit. Thus it is tempting to interpret spindle galaxies as galaxies with two orthogonal families of stable loop orbits (Tohline, Simonson \& Caldwell 1982, van Albada, Kotanyi \& Schwarzschild 1982). It would the follow that many S0 galaxies have triaxial potentials. Furthermore, the ring of a typical spindle galaxy rotates too slowly to follow the orientation of a bar in the visible galaxy, so any large-scale triaxiality of the potential would be generated by a triaxial halo.

This is an interesting line of argument, but two cautions are in order. (i) As Schweizer et al. (1983) have emphasized, the dynamical times of polar rings are very long, and the time required for nearly polar gas in an axisymmetric potential to settle to the equatorial plane can be very long. Hence it is not clear whether the observed rings really delineate families of stable loop orbits, or simply planes from which orbital decay is exceptionally slow. (ii) It is not clear that the differential precession, or libration periods of stellar orbits provide an adequate measure of the time required for a stream of gas to evolve from that orbit towards the relevant closed orbit. Convincing numerical simulations of this settling process, including realistic accounts of the action of viscosity and self-gravity, are not yet available, although studies have been published of the effects of self-gravity and viscosity taken in isolation.

Steiman-Cameron (1984) and Habe \& Ikeuchi (1985) have presented numerical calculations of the settling of gaseous annuli in axisymmetric and triaxial potentials. Steiman-Cameron models the gaseous annuli with a series of thin rings. He finds that the time required for gas to settle to a preferred plane depends sensitively on the difference in the free precession or libration periods of stellar orbits accross the annulus. By contrast, large changes in the coefficient of viscosity lead to only small changes in the settling time. For relatively low coefficients of viscosity, Steiman-Cameron's annuli, unlike observed rings, become strongly twisted. The models of Habe \& Ikeuchi employ extended particles. Hence they cannot simulate the low-viscosity regime. The annuli all settle to preferred planes in the appropriate precession or libration time.

Sparke (1984b) has recently discussed the effects of self-gravity on the evolution of a frictionless polar ring in an axisymmetric potential. She finds that self-gravity can stabilize a ring of realistic mass providing it warps towards the 
pole on the outside. This is exactly the reverse sense of warp to that predicted by assuming that the ring is made up of closed loop orbits in a tumbling triaxial potential. Sparke's work seems to be an important advance, which probably gives a truer account of the evolution of a real polar ring than do the calculations of Steiman-Cameron and Habe \& Ikeuchi.

2) From the shapes and velocities of rings we may determine the flattening of the halo potential (Schweizer et al.1983, Katz \& Richstone 1984). The flatter the combined galaxy/halo potential is, the more the ring will be elongated parallel to the galaxy's shortest axis, and the slower the stream will move over the pole. For favourable ring orientations, these effects could be used to determine one axis ratio of the halo potential:

(i) If in some galaxy one finds no velocity gradient perpendicular to the line in which a polar ring cuts the equatorial plane of the embedded galaxy, the latter line must lie in the plane of the sky. The ring's intrinsic shape could then be determined from the apparent axis ratios of the ring and the disk. Application of the epicycle approximaton to polar rings, reveals that the ring ellipticity determined in this way would be, for a flat circular-speed curve, equal to the ellipticity of the potential (although the ring is extended parallel to the ring's short axis).

(ii) If the ring is seen nearly edge on, the axis ratio $q_{\Phi}$ of the equipotential surfaces could be determined by comparing the peak radial velocity $v_{\text {ring }}$ in the ring with an estimate $v_{d i s k}$ of the circular speed at a similar radius in the underlying galactic disk: For this case the epicycle gives for a flat circular-speed curve, $q_{\Phi} \simeq\left(2 v_{\text {ring }}+\right.$ $\left.v_{d i s k}\right) / 3 v_{d i s k}$.

\section{Ripples}

In 1980 Malin \& Carter drew attention to the presence of sharp steps in the outer brightness profiles of some giant elliptical galaxies. They called these features shells, but I shall follow Schweizer $(1980,1983)$ in referring to them as ripples since they are almost certainly not generated by shells of stars.

The survey of Malin \& Carter (1983) has shown that on the order of $17 \%$ of isolated elliptical galaxies have ripples in their envelopes. Photometric observations (Carter, Allen \& Malin 1982) have revealed that ripples are generated by abrupt steps in stellar density. A single ripple never seems to envelope the whole galaxy. They tend to cluster along the apparent major axis, and to be interleaved in radius on opposite sides of the nucleus. Ripples have been detected as far out as $3 R_{e}$.

Quinn (1984) has investigated Schweizer's (1980) suggestion that ripples represent the debris of disk galaxies that have been eaten by a giant. This proposal hinges on the strong concentraton in phase space of the stars of any disk galaxy; in real space disk stars are concentrated to a plane, and at any point within this plane their velocity vectors cluster around the circular velocity. Hence the phase- 
space density of disk stars is appreciable only near a two-dimensional subspace of the full six-dimensional phase space. By Liouville's theorem, this concentration of the disk stars is not affected by the cannibalization of the disk by the giant. Consequently the projected image of the predator galaxy should subsequently be marked by regions of enhanced luminosity wherever we view this phase-space sheet edge-on.

The easiest way to picture the formation of high-density ripples around a giant galaxy is to imagine the fate of a low-mass disk that approaches a giant on a high-angular momentum orbit. The disk will be stretched and wrapped like a bandage around the predator. High surface densities will occur where the line of sight is tangent to this bandage. However, Quinn found that his test-particle models generated the most convincing ripples when a disk was dropped into the predator on a deeply plunging orbit. Beautiful ripples were then formed at the radial turning points of the resulting stellar orbits. Stars linger at these turning points, with the result that those orbits on which stars are currently at apocentre stand out prominently. This model of ripples accounts very naturally for the interleaving of the ripples on either side of the nucleus and for their tendency to occur along the apparent major axes of galaxies. If Quinn is correct in maintaining that ripples are generated by stars on very radial orbits and not by bandaging giants with disks, we should think of ripples as being generated by phase-space sheets rather than by real-space sheets of stars.

Ripples may soon prove very powerful probes of halo structure. The radial density profile could follow from the radii $r_{n}$ of successive ripples, these being the radii at which $n$ radial periods have elapsed since the victim fell in (Quinn 1984). The angular structure would follow from such things as whether pericentre distances are marked by reversed ripples near the centre, and whether there are ripples with angular structure characteristic of long-axis tubes in addition to ripples generated by boxes and short-axis tubes.

However, it will not be easy to test these predictions because the existing calculations of ripple formation are not very convincing, and it may not be easy to improve on them in the near future. The difficulty is that the entrapment of the victim by the tidal wave which the victim raises in the predator determines the final energy distribution of the former disk stars. Hence credible calculations cannot be performed with test-particle disks such as those employed by Quinn. On the other hand, full $n$-body calculations are not only much more expensive, but are, as Quinn (1984) discovered, also unlikely to produce nice ripples: Currently affordable $n$ body calculations are always contaminated by a degree of collisional relaxation (Norman, May \& van Albada 1985). This artificially fattens an otherwise thin phase-space stream of stars, and hence blurrs any ripples to which that stream gives rise. The solution to this problem would seem to require some cunning combination of the $n$-body and test-particle techniques.

However, I think triaxiality of the halo can help us to a qualitative understanding of why so many giant ellipticals have swallowed their victims from highly 
eccentric orbits: The fraction of phase space that is occupied by highly elongated orbits is simply higher in a triaxial potential than in an axisymmetric one. Consequently, if a halo is triaxial, the victim galaxy is likely to approach the predator on a highly eccentric orbit.

\section{References}

Aarseth, S.J. \& Binney, J.J., 1978. Mon. Not. Roy. astr. Soc., 185, 227.

Barnes, J., Goodman, J. \& Hut, P., 1985. Astrophys. J., 000, 000.

Barnes, J. \& White, S.D.M., 1984. Mon. Not. Roy. astr. Soc., 211, 753.

Bertin, G. \& Mark, J.W.-K., 1980. Astron. Astrophys., 88, 289.

Binney, J.J., 1976. Mon. Not. Roy. astr. Soc., 177, 19.

Binney, J.J., 1978. Mon. Not. Roy. astr. Soc., 183, 779.

Binney, J.J., 1981. Mon. Not. Roy. astr. Soc., 196, 455.

Binney, J.J. \& May, A., 1985. Mon. Not. Roy. astr. Soc., 000, 000.

Binney, J.J. \& Silk, J, 1978. Comm. Astrophys. Sp. Sci., 7, 139.

Carter, D., Allen, D.A. \& Malin, D.F., 1982. Nature, 295, 126.

Di Tullio, G.A., 1979. Astron. Astrophys.Suppl. Ser., 37, 591.

Efstathiou, G. \& Jones, B.J.T., 1979. Mon. Not. Roy. astr. Soc., 186, 133.

Fall, S.M. \& Efstathiou, G., 1980. Mon. Not. Roy. astr. Soc., 193, 189.

Gerhard, O.E., 1981. Mon. Not. Roy. astr. Soc., 197, 179.

Gerhard, O.E., 1983. Mon. Not. Roy. astr. Soc., 203, 19P.

Gunn, J.E., 1982. In: Astrophysical Cosmology,, eds H.A. Brück, G.V. Coyne \& M.S. Longair, pp 23 (Vatican: Pontif. Acad.).

Habe, A. \& Ikeuchi, S., 1985. Astrophys. J., 289, 540.

Heiligman, G. \& Schwarzschild, M., 1979. Astrophys. J., 233, 872.

Heisler, J., Meritt, D. \& Schwarzschild, M., 1982. Astrophys. J., 258, 490.

Hunter, C. \& Toomre, A., 1969. Astrophys. J., 155, 747.

Katz, N. \& Richstone, D.O., 1984. Astron. J., 89, 975.

Malin, D.F. \& Carter, D., 1980. Nature, 285, 643.

Malin, D.F. \& Carter, D., 1983. Astrophys. J., 274, 534.

Marthur, S.D., 1984. Mon. Not. Roy. astr. Soc., 211, 901.

Merritt, D. \& Aguilar, L., 1985. Mon. Not. Roy. astr. Soc., 000, 000.

Miller, R.H. \& Smith, B.F., 1981. Astrophys. J., 244, 33.

Mulder, W., 1983. Astron. Astrophys., 121, 91.

Negroponte, J. \& White, S.D.M., 1983. Mon. Not. Roy. astr. Soc., 205, 1009.

Norman, C.A., May, A., \& van Albada, T.S., 1985. Astrophys. J., 000, 000. 
Peebles, P.J.E., 1969. Astrophys. J., 155, 393.

Petrou, M., 1980. Mon. Not. Roy. astr. Soc., 191, 767.

Quinn, P.J., 1984. Astrophys. J., 279, 596.

Richstone, D.O. \& Potter, M., 1982. Nature, 298, 728.

Schechter, P.L. \& Gunn, J.E., 1978. Astron. J., 83, 1360.

Schechter, P.L., Sancisi, R., van Woerden, H. \& Lynds, C.R., 1984. Mon. Not. Roy. astr. Soc., 208, 111.

Schechter, P.L., Ulrich, M.-H. \& Boksenberg, A., 1984. Astrophys. J., 277, 531.

Schweizer, F., 1980. Astrophys. J., 237, 303.

Schweizer, F., 1983. In: IAU Symposium 100, Internal Kinematics and Dynamics of Galaxies, (Dordrecht: Reidel), pp. 319.

Schweizer, F., Whitmore, B.C. \& Rubin, V., 1983. Astron. J., 88, 909.

Sparke, L.S., 1984a. Astrophys. J., 280, 117.

Sparke, L.S., 1984b. Mon. Not. Roy. astr. Soc., 211, 911.

Sparke, L.S., 1985. Mon. Not. Roy. astr. Soc., 000, 000.

Steiman-Cameron, T.Y., 1984. Ph.D. Thesis, University of Indiana.

Tohline, J.E. \& Durisen, R.H., 1982. Astrophys. J., 257, 94.

Tohline, J.E., Simonson, G.F. \& Caldwell, N., 1982. Astrophys. J., 252, 92.

Toomre, A., 1983. In: IAU Symposium 100, Internal Kinematics and Dynamics of Galaxies, (Dordrecht: Reidel), pp. 177.

Tubbs, A.D. \& Sanders, R.H., 1979. Astrophys. J., 230, 736.

van Albada, T.S., Kotanyi, C.G. \& Schwarzschild, M., 1982. Mon. Not. Roy. astr. Soc., 198, 303.

White, S.D.M., 1979. Mon. Not. Roy. astr. Soc., 189, 831.

White, S.D.M. \& Rees, M.J., 1978. Mon. Not. Roy. astr. Soc., 183, 341.

Wilkinson, A. \& James, R.A., 1982. Mon. Not. Roy. astr. Soc., 199, 171. 


\section{DISCUSSION}

PEEBLES: You described how a disk misaligned with an aspheric halo will precess. But I reckon that the precession rate is a function of radius, so a stellar disk would end up being thick. This is not acceptable, isn't that right?

BINNEY: If gas comes in on a skew axis, the stars that form out of it do indeed precess around and form a thick disk. What is interesting, though, is the response of the pre-existing galaxy. It turns out that an adiabatic invariance enables this stuff to turn right over in the new potential and stay thin.

GUNN: My impression is that the warps one sees begin at about the place where disks become non-self-gravitating. Could you comment on that?

BINNEY: I think you would say that warping is just a transient phenomenon - the junk falling in temporarily doesn't have the proper orientation because we are looking far out in the halo. I think I would agree. But then warps don't last long unless you have steady infall.

CASERTANO: It is true that most warps seem to start where there is little star light, but the local neutral hydrogen density is enough for self-gravity to be important. Besides, there is at least one case (NGC 4565, see poster paper by Casertano, Sancisi and van Albada) where the stars also take part in the distortion.

VAN WOERDEN: On the matter of polar rings: W. van Driel and $I$ have mapped HI in 10 gas-rich S0 galaxies and $6 \mathrm{~s} 0 / \mathrm{a}^{\prime} \mathrm{s}$. Among these, 6 and 1 , respectively, have their HI in rings outside the optical body. At least three of these rings are polar. Hence the polar-ring phenomenon is quite frequent.

BINNEY: Perhaps these are SO's precisely because they have ceased to accrete material in their equatorial planes. Accretion over the pole is unlikely to be compatible with continued accretion in the equatorial plane, and spiral structure is then likely to die out.

WHITMORE: I would like to add a few items to the description of polar ring galaxies. About half of these systems actually have a thin ring rather than a broad annulus. In this case differential precession may be negligible. However, long-slit spectra show emission over a much wider region than the thin optical ring, suggesting that a disk is present. Perhaps some type of resonance phenomena results in star formation only at a certain radius, producing the thin ring. I hope theorists will address this question.

I would also like to report that one of the candidate polar ring galaxies reported by Schweizer, Whitmore, and Rubin (A.J., 88, 909, 1983) turns out to be an elliptical rather than an S0. This system is described in a poster paper. 
SCHWARZSCHILD: Earlier, Sancisi showed us one case where the HI was marvellously aligned but lopsided, meaning that phase mixing within the plane was not complete but that somehow orientation to the plane was well achieved. Can you do that with a highly flattened halo?

BINNEY: Yes, I think you can. If you rely on something like dynamical friction, then it is always the smallest component of the motion that's destroyed first. I don't know quantitatively how flat the halo would have to be.

RICHSTONE: James, can you expand on one of your earlier remarks? Why the preference for radial orbits in ripples and shells?

BINNEY: I think that triaxiality provides a very natural explanation, because it says that a very large portion of phase space consists of box orbits. Box orbits are all by definition extremely radial. In the transition from an axisymmetric to a triaxial potential, the most eccentric orbits go over into box orbits. So what used to take up a negligible proportion of phase space starts to take up a lot of phase space. I suspect that if you drop something into a triaxial halo even with a reasonable amount of angular momentum, it will still wind up in a box orbit - effectively, a zero-angular-momentum orbit.

LYNDEN-BELL: Assuming the Aarseth and Binney principle that anything that can happen does happen, would you not expect the barycenter of the halo to be initially offset from that of the visible galaxy? The initial sloshing of a galaxy within its halo may have interesting unsymmetrical consequences.

BINNEY: Gerhard (M.N.R.A.S., 203, 19P, 1983) and May, Norman and van Albada (Ap.J., in press) have found that n-body models sometimes continue oscillating for many dynamical times after violent relaxation would be expected to be complete. Hence it is indeed likely that halos, which must have very long dynamical times, are still shivering like cheap jellies. Perhaps a large-scale example of such a shiver is provided by the offset observed between the center of the Perseus $x$-ray source and the cooler $x$-ray source centered on NGC 1275 (BranduardiRaymont et a1., Ap.J., 248, 55, 1981).

OSTRIKER: Do you believe that either 1) the shapes of x-ray isophotes, or 2) the fact that the surface density needed for gravitational lenses does not coincide with the surface light density give evidence for triaxiality of dark matter accumulations?

BINNEY: Certainly I have long been an enthusiast for using $x$-rays to determine the shapes of clusters of galaxies; Sarazin yesterday mentioned the work Strimpel and I did a few years ago to develop this approach. It would certainly be interesting to apply that method to ellipticals. I have not thought about gravitational lenses, but it is tempting to blame some of the problems Turner discussed yesterday on the triaxiality of halos. 